\title{
Bildgebung transmesokolischer innerer Hernien nach retrokolischer Roux-Y-Rekonstruktion: Tipps und Tricks für Radiologen
}

\author{
Imaging of Transmesocolic Internal Hernias Following Retrocolic \\ Roux-Y Reconstruction: Tips and Tricks for Radiologists
}

Autoren

Institut
T. Meissnitzer, M. W. Meissnitzer, R. Forstner, K. Hergan

Universitätsinstitut für Radiologie, Landeskrankenhaus Salzburg

\section{Key words \\ - abdomen \\ - CT \\ - hernia}

eingereicht 22.9.2012

akzeptiert 7.1.2013

Bibliografie

DOI http://dx.doi.org/

10.1055/s-0032-1330740

Online-Publikation: 14.3.2013

Fortschr Röntgenstr 2013; 185:

419-427 @ Georg Thieme

Verlag KG Stuttgart · New York ·

ISSN 1438-9029

Korrespondenzadresse Dr. Thomas Meissnitzer

Department of Radiology, University Hospital of Salzburg Müllner Hauptstrasse 48

5020 Salzburg

Österreich

Tel.: ++43/662/448258987

Fax: ++43/662/44823964

thomas.meissnitzer@A1.net

\section{Zusammenfassung}

$\nabla$

Intestinale Passagestörungen werden zwar selten durch innere Hernien hervorgerufen, dennoch können nach retrokolischer Roux-y-Rekonstruktion im Rahmen onkologischer oder bariatrischer Magenoperationen transmesokolische innere Hernien auftreten. Begünstigend wirkt die postoperative Abnahme mesenteriellen Fettgewebes bei gleichzeitig erhaltener Lageverschieblichkeit des Darmes nach der heutzutage bevorzugten laparoskopischen Operationstechnik. In der Bildgebung postoperativer innerer Hernien finden sich die spezifischen CT-Befunde einer segmentalen intestinalen Obstruktion analog zu sämtlichen differenzialdiagnostisch relevanten Entitäten. Alleinig die exakte Analyse der pathognomonisch veränderten Topografie der Peritonealhöhle, die auch durch die Mesenterien definiert ist, gestattet dem Radiologen die Abgrenzung zu Briden als wichtigste Differenzialdiagnose. Basierend auf einfach und eindeutig aufzufindenden anatomischen Landmarken werden im Folgenden die diagnostischen Kriterien postoperativer transmesokolischer innerer Hernien in der Computertomografie herausgearbeitet. Besonderes Augenmerk wird auf das Mesocolon transversum und dessen Rolle als Kompartmentbegrenzung innerhalb der Peritonealhöhle gelegt. Zusammenfassend wird eine Checkliste für die tägliche radiologische Praxis präsentiert.

\section{Einleitung}

Während die chirurgische Behandlung der Ulkuskrankheit zugunsten der medikamentösen Therapie stark in den Hintergrund getreten ist, stehen heute onkologische und bariatrische Operationen am Magen im Vordergrund [1, 2]. Gängigstes Verfahren zur Wiederherstellung der Kontinuität des Verdauungstrakts ist dabei die Roux-Y-Rekons-

\section{Abstract \\ $\nabla$}

Among all entities causing intestinal obstruction, internal hernias are rare. However, after retrocolic laparoscopic Roux-Y reconstruction, transmesocolic internal hernias may occur. The loss of mesenteric fat favors the formation of transmesocolic internal hernias, which are the most common type of acquired internal hernias. CT findings of segmental intestinal obstruction are similar in all underlying diseases including peritoneal adhesions, which are the most important differential diagnosis to postoperative internal hernias. Since internal hernias typically alter spatial relationships within the peritoneal cavity, precise analysis of intraperitoneal topography is the most important clue to differentiate internal hernias from peritoneal adhesions. Based on readily identifiable anatomic reference points and structures, so-called landmarks, specific features of internal hernias in CT imaging are outlined. Particular attention is paid to mesenteries, because they define compartments within the peritoneal cavity. Focusing on transmesocolic internal hernias, the anatomy of the mesocolon transversum is described in detail. Finally, we present a checklist facilitating the diagnosis of internal hernias in everyday practice. truktion, bei der einerseits eine Jejunumschlinge mit dem Restmagen bzw. nach totaler Gastrektomie mit dem Ösophagus verbunden wird (=abführende Schlinge) und andererseits die die Galle und den Pankreassaft ableitende orale Jejunumschlinge (= biliodigestive Schlinge) mit der abführenden Schlinge Y-förmig anastomosiert wird [3]. Die laparoskopische Operationstechnik wird heute gegenüber dem offen chirurgischen Vorgehen 
bevorzugt, da kardiopulmonale Komplikationen, postoperative Wundheilungsstörungen, Bauchwandhernien und peritoneale Adhäsionen seltener auftreten [3 -7].

Typische Komplikationen dieser chirurgischen Methode umfassen Naht- und Anastomoseninsuffizienzen, postoperative Infekte, Blutungen sowie intestinale Obstruktionen. Erstere können mittelund längerfristig zu einem Therapieversagen mit erneuter Gewichtszunahme führen (durch die Ausbildung einer Fistel zwischen oralem Magenpouch und analem Restmagen), des Weiteren erfordern Abszesse und Blutungen ein unmittelbares chirurgisches oder interventionell-radiologisches Eingreifen [3, 5-8].

Gründe für intestinale Obstruktionen, die in Studien von DeMaria et al., Schauer et al. und Higa et al. in bis zu 11,7\% nach laparoskopischen Roux-Y-Rekonstruktionen auftreten, sind Anastomosenstenosen an der Gastrojejunostomie oder Jejeunojejunostomie, Einschnürungen des Darmes an mesenteriellen Durchtrittspforten, Adhäsionen sowie äußere und innere Hernien $[6,7,9,10]$. Mit bis zu 4,5\% ist die Inzidenz innerer Hernien nach laparoskopischer Rekonstruktion höher als nach offenem Vorgehen [11, 12].๑ Tab. 1 fasst die genannten Komplikationen zusammen.

Diffuse Bauchschmerzen, Nausea und Erbrechen sind die klinischen Symptome einer intestinalen Obstruktion. Laborchemisch weisen Hämatokriterhöhung, Leukozytose, Elektrolytentgleisung sowie LDH- und Laktaterhöhung auf einen Ileus hin [13]. Weder die Klinik noch das Labor gestatten einen Rückschluss auf die zugrunde liegende Ursache der Darmpassagestörung.

$\mathrm{Zu}$ beachten ist, dass innere Hernien klinisch asymptomatisch sein können. Diese zu erkennen, ist auch für den Radiologen eine besondere Herausforderung, da sie bei in der Regel fehlender Darmdilatation nur durch eine veränderte Lage von Teilen des Bauchhöhleninhalts zu diagnostizieren sind.

Die Computertomografie ist neben der Durchleuchtungsuntersuchung aufgrund der ubiquitären Verfügbarkeit und der kurzen Untersuchungsdauer die bevorzugte Untersuchungsmodalität zur Abklärung abdomineller Beschwerden [14].

Dieser Übersichtsartikel beschäftigt sich mit der CT-Diagnostik von inneren Hernien nach Roux-Y-Operationen unter besonderer Berücksichtigung des transmesokolischen Typs. Ein zeitgemäßes Untersuchungsprotokoll umfasst die Rohdatenakquisition zumindest in der portalvenösen Phase nach intravenöser Kontrastmittelapplikation mittels Multislice-Scanner, um daraus für die Befundung nützliche Rekonstruktionen herstellen zu können. Die perorale Gabe von Wasser als negatives Kontrastmittel vereinfacht die Beurteilung der Struktur und der Perfusionssituation von Magen und Darm entscheidend, wird jedoch gerade von diesen Patienten oft nicht toleriert. Da in diesen Fällen meist eine Darmpassagestörung vorliegt, ist hier bereits eine negative Darmlumenkontrastierung durch den retinierten Darminhalt gegeben. Transmesokolische Hernien treten ausschließlich nach retrokolischem Hochzug der efferenten Jejunumschlinge auf (siehe nachfolgende Beschreibung über die Prinzipien der Roux-Y-Rekonstruktion). Im Vergleich zum antekolischen Hochzug wird als Vorteil der retrokolischen Elevation die geringere Spannung an der Ösophago- bzw. Gastrostomie angesehen, als Nachteil das bis zu viermal höhere Risiko für das Auftreten innerer Hernien. In der bariatrischen Chirurgie wird daher zunehmend der antekolische Weg gewählt [11].

Die Detektion transmesokolischer Hernien wird basierend auf anatomischen Landmarken herausgearbeitet, wobei besonderes Augenmerk auf die Mesenterien gelegt wird.

Zusammenfassend wird in Form einer Checkliste ein möglicher Diagnosepfad vorgestellt, um Radiologen die Erkennung innerer
Tab. 1 Komplikationen nach laparoskopischer Roux-Y-Rekonstruktion. Die Häufigkeitsangaben stellen Durchschnittswerte dar [6, 7, 9, 10]. Nach retrokolischem Jejunumhochzug ist das Risiko für die Entstehung einer inneren Hernie um den Faktor 4 höher im Vergleich zur antekolischen Elevation [10].

\begin{tabular}{ll} 
Naht- u. Anastomoseninsuffizienzen & $2,5-5,1 \%$ \\
\hline Ulzera an der Gastrojejunostomie & $1-5,1 \%$ \\
\hline gastrointestinale Obstruktionen & $8,7-11,7 \%$ \\
- Innere Hernien & $1,8-4,5 \%$ \\
- Bauchwandhernien (Porthernien) & $0,7 \%$ \\
\hline Wundinfekte & $1,1-8,7 \%$ \\
Blutungen & $0,25-3,3 \%$
\end{tabular}

Hernien und deren Komplikationen zu erleichtern. Nachfolgender Haupttext und die Checkliste sind konkordant aufgebaut.

\section{Prinzipien der Roux-en-Y-Rekonstruktion}

$\nabla$

Zunächst erfolgt die Gastrektomie (z. B. bei onkologischer Indikation) oder die Magenverkleinerung (z.B. bariatrische Chirurgie). Letztere erfolgt im Rahmen der Adipositaschirurgie etwa parallel zur kleinen Magenkurvatur, sodass ein kleiner Magenpouch (Volumen $10-30 \mathrm{ml}$ ) und ein größerer Restmagen entstehen $[15,16]$. Nach Jejunotomie $25-50 \mathrm{~cm}$ anal der Treitz-Flexur wird die abführende Schlinge mit dem Ösophagus end-zu-end bzw. mit dem Restmagen end-zu-seit isoperistaltisch anastomosiert. Dazu muss diese efferente Jejunumschlinge entweder ante- oder retrokolisch in das linke Epigastrium eleviert werden. Ein retrokolischer Hochzug erfordert die Inzision des Mesocolon transversum. Die Weite dieser transmesokolischen Durchtrittsöffnung ist insofern kritisch, als einerseits eine zu kleine Öffnung die efferente Schlinge komprimiert („mesocolic tunnel stenosis“) und andererseits ein zu weiter Spalt eine potenzielle Bruchlücke für innere Hernien darstellt [3]. Eine Erweiterung dieses Gekröseschlitzes wird auch durch die postoperative Gewichtsabnahme mit Rückbildung mesenteriellen Fettgewebes begünstigt [12]. Die den Restmagen, das Duodenum (und damit Leber und Pankreas) sowie das orale Jejunum drainierende biliodigestive Schlinge wird mit der efferenten Schlinge $40-150 \mathrm{~cm}$ distal der Gastrobzw. Ösophagojejunostomie seit-zu-seit, Y-förmig verbunden ( $\bullet$ Abb. 1a-c) [3, 8]. Als typische Lage der Jejunojejunostomie wird in der Literatur der linke Mittelbauch in Höhe mittleres Nierendrittel angegeben [15].

Im Falle der Adipositaschirurgie fußt die Rationale dieses Vorgehens auf zwei Prinzipien:

Die Magenverkleinerung reduziert dessen Fassungsvermögen (restriktives Prinzip).

Die Umgehung des Restmagens, des Duodenums und des oralen Jejunums („Roux-Y-Magenbypass“) verkürzt (funktionell) die absorbierende Darmwegstrecke (malabsorptives Prinzip) durch das Fehlen der Galle und des Pankreassafts in der efferenten Schlinge. Im Gegensatz zu rein magenverkleinernden Verfahren (Magenband, horizontale bzw. vertikale Gastroplastie) resultiert aus der Kombination eines restriktiven mit einem malabsorptiven Verfahren ein nachhaltiger und dauerhafter Gewichtsverlust sowie eine wirksame Behandlung der mit der Adipositas assoziierten Komorbiditäten wie Diabetes, Hypertonie, Schlafapnoe-Syndrom, Hypoventilation und Hyperlipidämie [3, 4]. Über die Länge des Darmes anal der Jejunojejunostomie lässt sich das Ausmaß der Gewichtsabnahme in einem gewissen Maß beeinflussen, da die Nahrungsabsorption die Anwesenheit von Verdauungsenzymen 


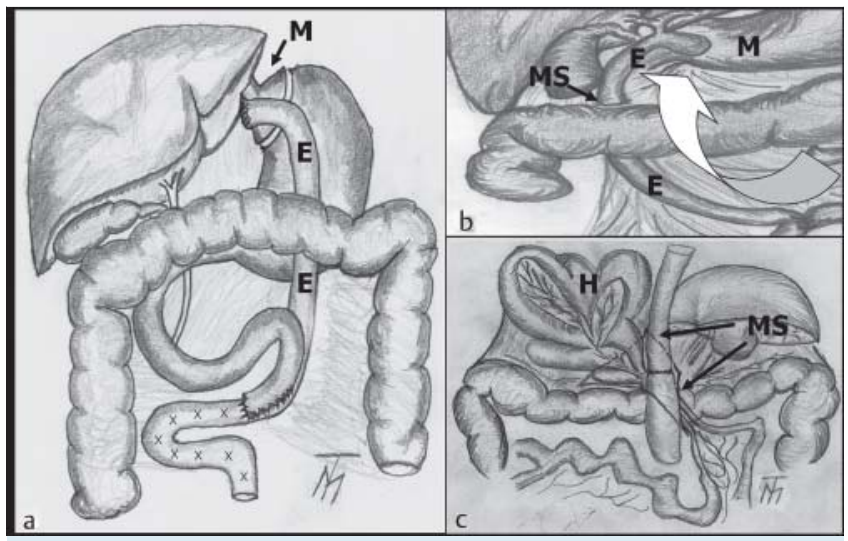

Abb. 1 a-c Schematische Darstellung des postoperativen Situs nach RouxY-Rekonstruktion. a Die efferente Jejunumschlinge (E) ist retrokolisch zum Restmagen (M) hochgezogen. Der Dünndarm anal der Jejunojejunostomie ist mit „x“ markiert. Hier kann bei eingeleiteten Verdauungsenzymen die Nahrungsabsorption wieder erfolgen. Je weiter anal die Jejunojejunostomie angelegt wird, umso kürzer ist der absorbierende Dünndarmabschnitt. b, c (Ansicht von dorsal): Hervorgehoben ist jeweils die chirurgisch geschaffene Öffnung im Mesocolon transversum für die efferente Schlinge. Dieser Mesenterialschlitz (MS) fungiert als Bruchring einer transmesokolischen Hernie $(\mathrm{H})$, deren Entstehung durch den postoperativen Gewichtsverlust mit konsekutiver Erweiterung desselben begünstigt wird.

voraussetzt. In der $\bullet \mathbf{A b b} \mathbf{1} \mathbf{a}$ ist dies schematisch dargestellt. Um Folgen eines Malabsorptionssyndroms wie Leber- und Niereninsuffizienz, Cholelithiasis, Urolithiasis, Osteoporose und Arthrosen zu verhindern, ist jedoch die Länge des umgangenen Verdauungswegs limitiert $[3,8,17]$.

Mit der Zunahme der Zahl laparoskopischer Operationen hat sich das Spektrum der den postoperativen Darmobstruktionen zugrunde liegenden Pathologien von den peritonealen Adhäsionen bzw. Briden hin zu den inneren Hernien verschoben [12]. Die im Gegensatz zum offenen Vorgehen geringer ausgeprägten peritonealen Adhäsionen und die dadurch erhaltene Verschieblichkeit der Darmschlingen werden als Gründe hierfür angeführt. Die postoperative Abnahme des mesenteriellen Fettgewebes erleichtert ihrerseits die Darmverschieblichkeit. Die Rückbildung des peritonealen Fettgewebes begünstigt auch die Erweiterung der transmesokolischen Durchtrittsöffnung für die hochgezogene Jejunumschlinge und damit die Entstehung einer transmesokolischen Hernie $[11,15,16]$.

Um postoperative Hernien diagnostizieren zu können, ist die Kenntnis der Anatomie des postoperativen Situs unentbehrliche Voraussetzung, insbesondere wenn zum Untersuchungszeitpunkt kein Ileus vorliegt. Diese Fälle stellen eine besondere Herausforderung dar, da die Detektion einer inneren Hernie nur über die Analyse der veränderten Topografie des verlagerten Bauchhöhleninhalts in Relation zu präexistenten anatomischen Bezugspunkten (im Folgenden als Landmarken bezeichnet) gelingt. Das vorgestellte Konzept der Landmarken ist an die Ausführungen von Takeyama et al. und Martin et al. angelehnt und wurde von den Autoren für das vorliegende Thema adaptiert und ergänzt $[18,19]$.

\section{Landmarken}

$\nabla$

Die anatomische Zuordnung abdomineller Strukturen, insbesondere von Darmabschnitten, basiert kurz gefasst auf 3 Säulen:
Konfiguration (u.a. Kerckring-Falten, Haustrierung), Lage innerhalb der Bauchhöhle (u. a. laterale Anteile, linker Ober- bzw. Mittelbauch) und - mit Letzterer unmittelbar in Zusammenhang stehend - der intestinalen Gefäßversorgung. Die die verschiedenen Darmabschnitte versorgenden Gefäße erreichen diese nach Abgang aus der Bauchaorta via Mesenterien, die somit die „Gefäßstiele“ des Darmes bilden und - im Gegensatz zum parietalen Peritoneum an den Wänden der Bauchhöhle - ein Teil des viszeralen Peritoneums sind. Daraus leitet sich auch das Konzept der „Leitgefäße“ in der abdominellen Radiologie ab, das die exakte Zuordnung und Bezeichnung des einzelnen Mesenteriums über die Identifizierung des darin verlaufenden Gefäßes ermöglicht, da das Mesenterium selbst in der Computertomografie in der Regel nicht sichtbar bzw. nicht von den (teils ebenfalls fettgewebsisodensen) Nachbarstrukturen abgrenzbar ist [19, 20].

\section{Landmarken am Gastrointestinum}

Als Ergebnis einer regulären embryonalen Entwicklung der Darmanlage liegen der Magen und die Flexura duodenojejunalis im linken Oberbauch, das Coecum in der rechten Fossa iliaca. Diese 3 Landmarken sind vom befundenden Radiologen obligat aufzusuchen ( $\bullet$ Abb.2a) [21].

\section{Landmarken, die von Mesenterien gebildet werden}

Da die Darmschlingen und damit auch die sie versorgenden (viszeralen) Mesenterien in gewissem Umfang mobile Strukturen innerhalb der Bauchhöhle sind, werden Lage und Verlauf der viszeralen Mesenterien anhand ihrer ortskonstanten Ansatzlinien an der Peritonealhöhlenwand definiert [22]. Darmschlingen mit breitem und langem Mesenterium bei gleichzeitig kurzer Ansatzlinie stellen besonders lagevariable Strukturen dar und sind damit prädisponiert für eine Herniation.

Für die Diagnostik postoperativer innerer Hernien ist die Anatomie der Mesenterien insofern von immanenter Bedeutung, da sie die Peritonealhöhle in definierte, kommunizierende Kompartimente unterteilen. Physiologischerweise liegen die einzelnen Darmabschnitte in bestimmten peritonealen Kompartimenten und überschreiten deren Grenzen nicht.

Da das Mesocolon transversum jenen Teil der Peritonealhöhle nach kranial begrenzt, der Darmschlingen enthält, ist es für die Erkennung postoperativer transmesokolischer Hernien von besonderer Bedeutung ( $\bullet$ Abb. 2c, d).

\section{Landmarken, die von mesenteriellen Gefäßen gebildet werden}

Die Mesenterialgefäße, die es Radiologen ermöglichen, Mesenterien im Oberbauch anatomisch zuzuordnen, sind in der überwiegenden Mehrzahl Äste der Arteria u. Vena mesenterica superior $(\bullet$ Abb. 2a, b). Als deren Äste definieren die A. u. V. colica media - neben dem von ihnen versorgten Colon transversum - die Schnittbildanatomie des Mesocolon transversum ( $\bullet$ Abb. 2c, d). Von den übrigen Mesenterialgefäßen sind in Hinblick auf die hier abgehandelten postoperativen inneren Hernien die Hauptstämme von A. und V. mesenterica superior sowie die V. mesenterica inferior von Bedeutung. Da der Bruchinhalt immer eine Raumforderung in der Peritonealhöhle darstellt und damit eine Verlagerung von präexistenten Strukturen bedingt, ist das Verlagerungsmuster dieser Leitgefäße entscheidend für die Erkennung einer inneren Hernie. Multiplanare Reformationen (MPRs), Curved planar Reformations (CPRs) oder Maximum-Intensitätsprojektionen (MIPs) sind entscheidende Tools in der modernen CT-Diagnostik, um einerseits diese Leitstrukturen aufzufinden und um anderer- 


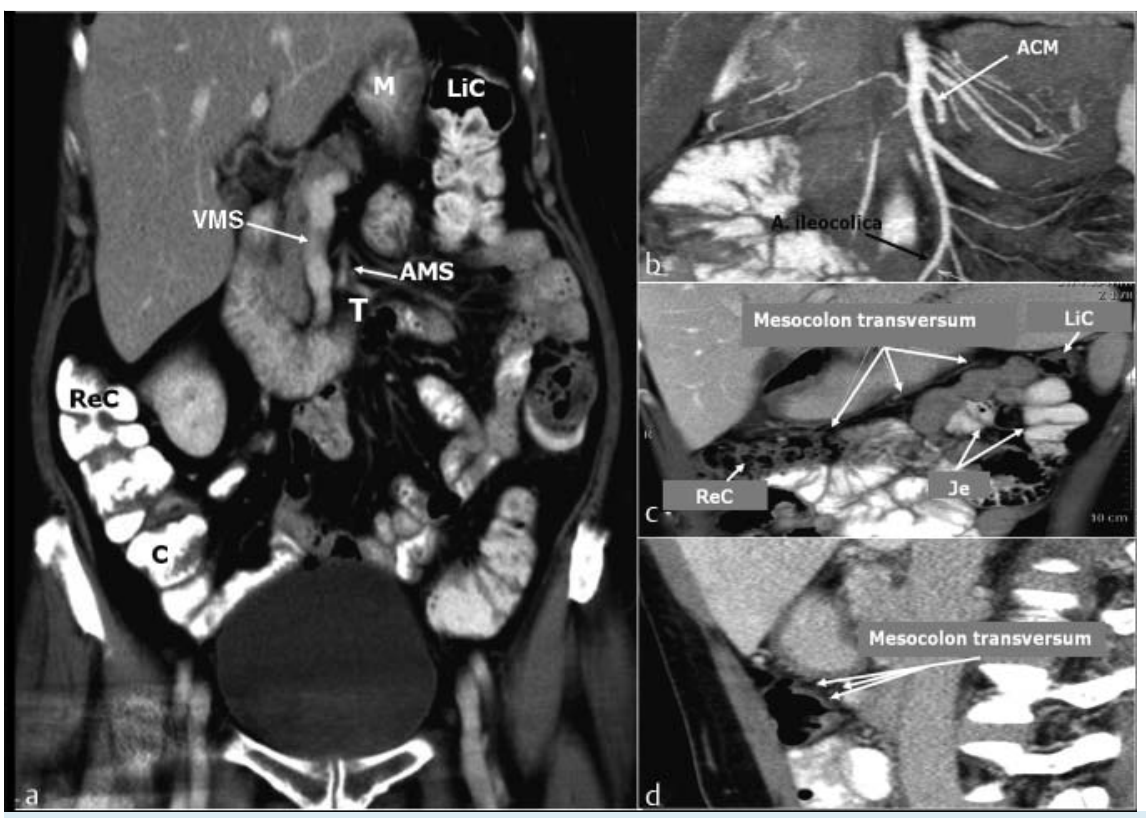

Abb. 2 a-d Obligat zu identifizierende Landmarken in der Abdomen-CT. a, b Landmarken am Gastrointestinum sowie an den Mesenterialgefäßen (MPR, koronar) mit orthotoper Lage des Magens (M) und der Treitz-Flexur (T) im linken Epigastrium sowie des Coecums (C) im rechten Unterbauch. Konsekutiv regelrechte Lagebeziehung zwischen Arteria (AMS) u. Vena mesenterica superior (VMS). Die MIP-Rekonstruktion der AMS in b erlaubt eine übersichtliche Darstellung ihres Verlaufs, die A. colica media (ACM) ist in dieser Rekonstruktion nur kurzstreckig am Abgang erfasst. Die AMS läuft in die A. ileocolica aus. Landmarken, die von Mesenterien gebildet werden wie das Mesocolon transversum, welches in der CT anhand der Vasa colica media und des zugehörigen Colon transversum zu identifizieren ist c, $\mathbf{d}$. Dessen Ansatzlinie quert ausgehend von der rechten Colonflexur (ReC) die retroperitoneal liegende Pars descendens duodeni sowie das Caput pancreatis in seinem kaudalen Drittel und verläuft nach Überschreiten der Medianebene entlang des Unterrands von Corpus und Cauda pancreatis in das linke Epigastrium, um hier an der linken Kolonflexur (LiC) zu enden. Die CPR der c zeigt das Mesocolon transversum in koronarer Ansicht, die $\mathbf{d}$ als MPR in der Sagittalen. Jejunumschlingen (Je).
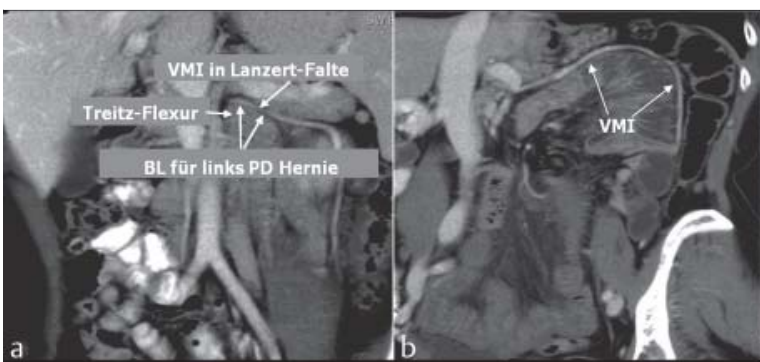

Abb.3 a-c Reguläre Anatomie der äußeren Pfortaderwurzeln a, typische Verlagerung der $\mathbf{V}$. mesenterica inferior $\mathbf{b}$ sowie angulierter Verlauf der Mesenterialgefäßäste als typischer Befund einer inneren Hernie c, CPRDarstellungen. Typische Anatomie der äußeren Pfortaderwurzeln mit Aszension der V. mesenterica inferior (VMI) in der Lanzert-Falte, welche die potenzielle Bruchlücke (BL) der linksseitigen paraduodenalen (PD) Hernie darstellt a, diese ist der häufigste Typus einer kongenitalen inneren Hernie. Typische Verlagerung der VMI nach links lateral b. Angulierter Verlauf der

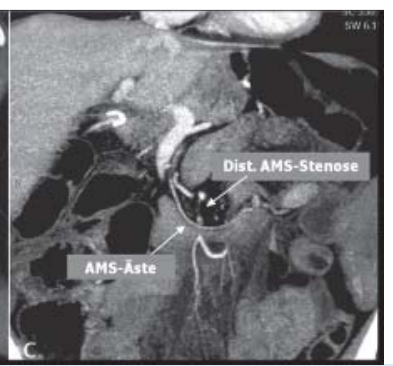

jejunalen A.-mesenterica-superior(AMS)-Äste im Fall einer inneren Hernie. Zusätzlich sind die ostealen A.- und V.-mesenterica-superior-Hauptstämme als Ausdruck der Raumforderung nach rechts verdrängt, die Stenose des distalen AMS-Stammes ist ebenfalls eine direkte Folge der Raumforderung im Oberbauch c. Entgegen dem physiologischen Descensus aszendieren die jejunalen AMS-Äste in das linke Epigastrium und markieren damit die Richtung der Herniation tributärer Strukturen (c, der gekrümmte Pfeil markiert die Herniationsrichtung). seits häufige anatomische Varianten erkennen zu können. In - Abb. 3a ist die klassische Anatomie der äußeren Pfortaderwurzeln dargestellt, in $\mathbf{A b b}$. $\mathbf{3 b}$ die typische Verlagerung der V. mesenterica inferior durch eine transmesokolische Hernie.

Vor allem in Hinblick auf die Detektion innerer Hernien ist nicht nur die Anatomie der genannten Gefäßhauptstämme, sondern auch die ihrer peripheren Äste von besonderem Interesse, da ein atypischer, z. B. angulierter oder torquierter Verlauf der peripheren Mesenterialgefäßaufzweigungen als wichtiger diagnostischer „Fingerzeig“ gilt ( $\bullet$ Abb. 3c).

\section{CT-Befunde postoperativer transmesokolischer Hernien}

$\nabla$

\section{Lage des Darmes}

Per definitionem handelt es sich bei einer postoperativen transmesokolischen Hernie um eine Verlagerung von Dünndarmschlingen in den Oberbauch (in der Regel in das linke Epigastrium), wobei sie durch den iatrogen angelegten Spalt im Mesocolon transversum hindurchtreten, durch den auch die efferente Jejunumschlinge zum Ösophagus bzw. zum Restmagen hochgezogen wurde (๑ Abb. 4a-c). 


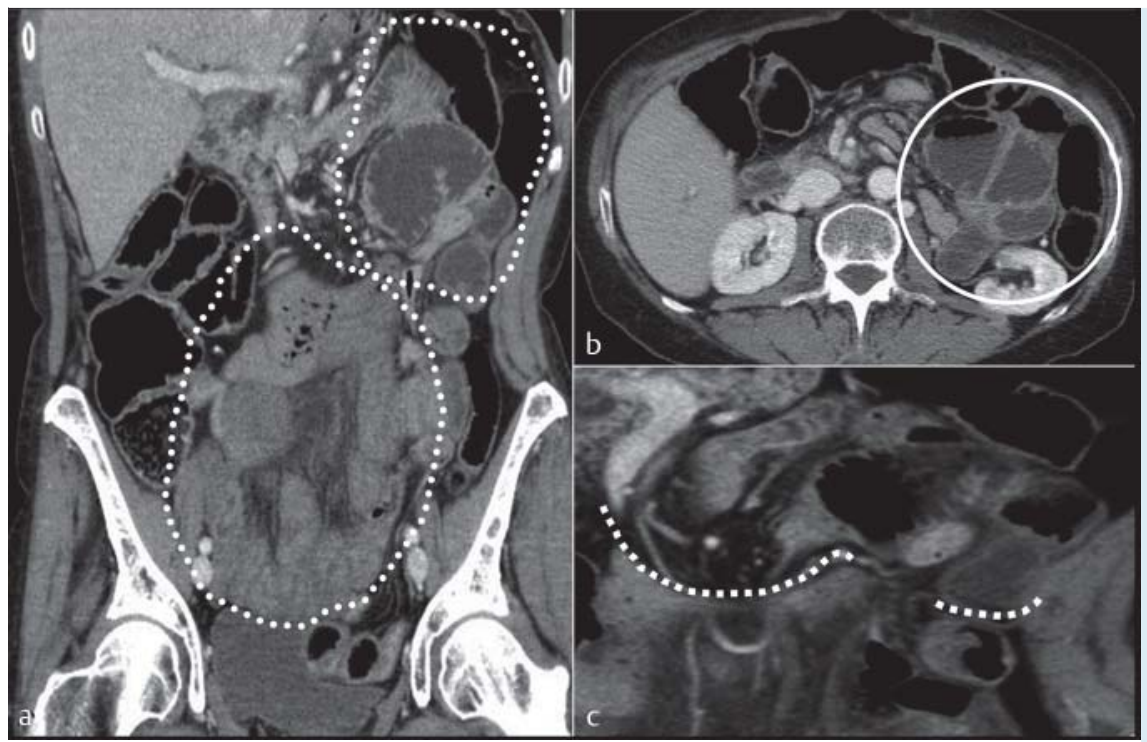

Abb.4 a-c Typische Befunde einer transmesokolischen inneren Hernie. Angulierte koronare MPR zur übersichtlichen Darstellung einer postoperativen transmesokolischen Hernie a. Die verlagerten, dilatierten Dünndarmschlingen liegen im linken Epigastrium. Durch die Konvergenz der Darmschlingen und der sie versorgenden Mesenterien in Richtung Bruchring im Mesocolon transversum beschreibt die strichlierte umhüllende Linie eine Achterfigur, diese tritt durch die begleitende venöse Stauung (Abb. 8) deutlicher hervor. Auch das pilzähnliche Bild („Mushroom-sign“) der hernierten Dünndarmschlingen mit den sie versorgenden Mesenterien ist damit zu erklären. Axialschnitt durch den Oberbauch zur Darstellung der hernierten, dilatierten Dünndarmschlingen (b, ringmarkiert). Koronarschnitt zur Darstellung des Bruchrings im Mesocolon transversum. Die strichlierte Linie markiert die Ansatzlinie des Mesocolon transversum, die Linienunterbrechung zeigt den Bruchring $c$.

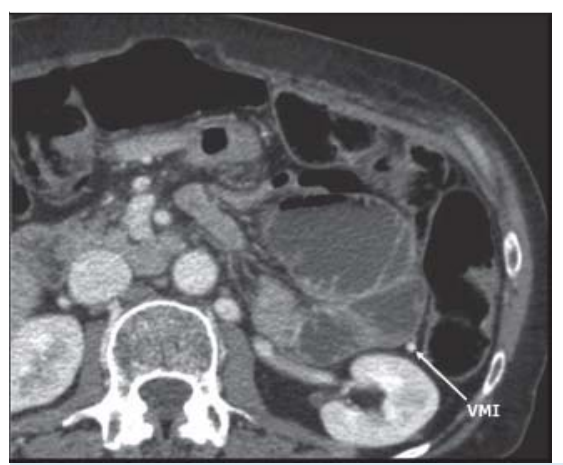

Abb.5 Fehlender Bruchsack einer transmesokolischen Hernie. Vergleichbar mit einer kongenitalen linksseitigen paraduodenalen Hernie kommt es zur Verlagerung der V. mesenterica inferior (VMI, pfeilmarkiert). Der fehlende Bruchsack im Fall einer postoperativen transmesokolischen Hernie (dieser würde sich als zarte lineare Verdichtung im V. mesenterica inferiorVerlauf demarkieren) gestattet jedoch eine Differenzierung (Axialschnitt durch den Oberbauch).

Die hernierten Dünndarmschlingen bilden somit neben dem verlagerten Gekrösefett den Bruchinhalt, der Bruchring ist der o.g. Spalt im Mesocolon transversum. Da es im Gegensatz zu angeborenen inneren Hernien zu keiner Aussackung des Peritoneums kommt, weisen transmesokolische Hernien keinen Bruchsack auf ( $\bullet$ Abb.5). Der fehlende Bruchsack gestattet somit die differenzialdiagnostische Abgrenzung von einer (kongenitalen) linksseitigen paraduodenalen Hernie [19]. Die $\bullet$ Tab. 2 vergleicht die CT-Befunde der transmesokolischen mit jenen der linksseitigen paraduodenalen Hernie.

Bei einer transmesokolischen Herniation von Dünndarmschlingen überschreiten diese die kraniale Begrenzung des „Darmbauchs“, also jenes Teiles der Peritonealhöhle, der physiologischerweise Darmschlingen enthält. Diese kraniale Begrenzung

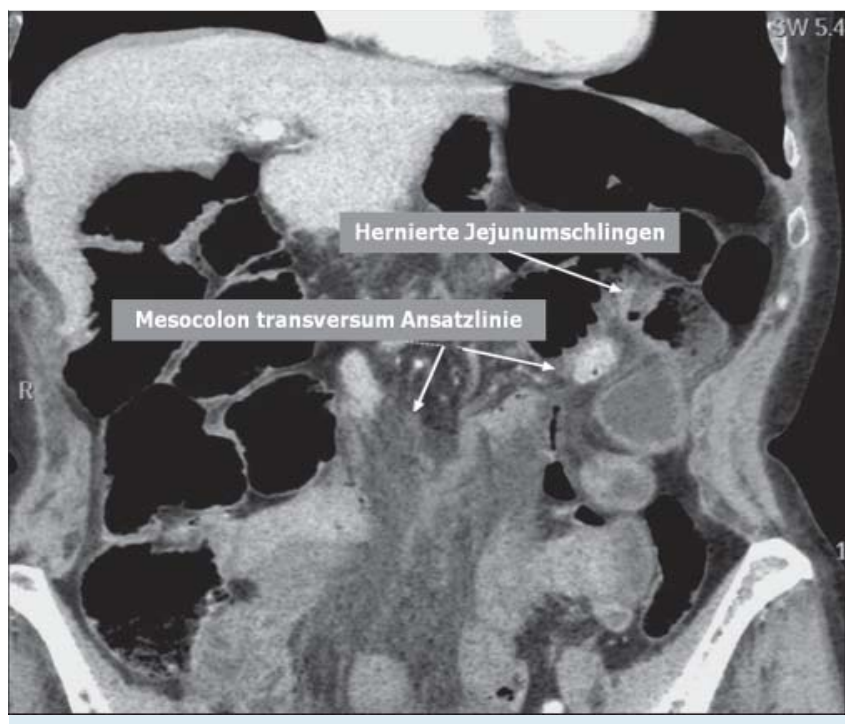

Abb. 6 Räumliche Beziehung zwischen hernierten Dünndarmschlingen und Ansatzlinie des Mesocolon transversum (CPR). Als typischer Befund einer transmesokolischen Hernie liegen die verlagerten Dünndarmschlingen cranial der Ansatzlinie des Mesocolon transversum. Das Mesocolon transversum stellt die kraniale Begrenzung jenes Teiles der Peritonealhöhle dar, der physiologischerweise Darmschlingen enthält.

des Darmbauchs ist durch die Ansatzlinie des Mesocolon transversum festgelegt, entsprechend ihrem Verlauf markiert somit der Unterrand des Pankreas diese Grenze. Abb. 6 veranschaulicht die räumliche Beziehung zwischen den verlagerten Dünndarmschlingen und der Ansatzlinie des Mesocolon transversum. Diese Überschreitung der kranialen Peritonealhöhlenbegrenzung durch die hernierten, im Epigastrium gruppiert liegenden Darmschlingen („Clustered loops“) konnten Reddy et al. bei allen ihrer 27 untersuchten Patienten mit transmesokolischen Hernien nach retrokolischem Magenbypass nachweisen [15].

Dorsal der Arteria mesenterica superior liegende Dünndarmschlingen (zusätzlich zur Pars horizontalis duodeni) können ebenfalls auf eine postoperative innere Hernie hinweisen („Small bowel behind superior mesenteric artery“) [16, 23]. 


\begin{tabular}{lllll} 
& Bruchlücke & Bruchinhalt (Lage) & Bruchsack & Leitgefäß \\
\hline $\begin{array}{l}\text { transmesokolische } \\
\text { Hernie }\end{array}$ & $\begin{array}{l}\text { transmesokolischer } \\
\text { Schlitz (iatrogen) }\end{array}$ & $\begin{array}{l}\text { Darmschlingen } \\
\text { (obligatorisch kranial d. } \\
\text { Mesocolon transversum) }\end{array}$ & nicht vorhanden & $\begin{array}{l}\text { V. mesenteri- } \\
\text { ca inferior }\end{array}$ \\
$\begin{array}{l}\text { links paraduodenale } \\
\text { Hernie }\end{array}$ & Lanzert-Falte & Darmschlingen & $\begin{array}{l}\text { Peritoneum d. } \\
\text { Lanzert-Grube }\end{array}$ & $\begin{array}{l}\text { V. mesenteri- } \\
\text { ca inferior }\end{array}$ \\
\hline
\end{tabular}

Tab. 2 Differenzialdiagnose postoperative transmesokolische Hernie versus kongenitale linksseitige paraduodenale Hernie.

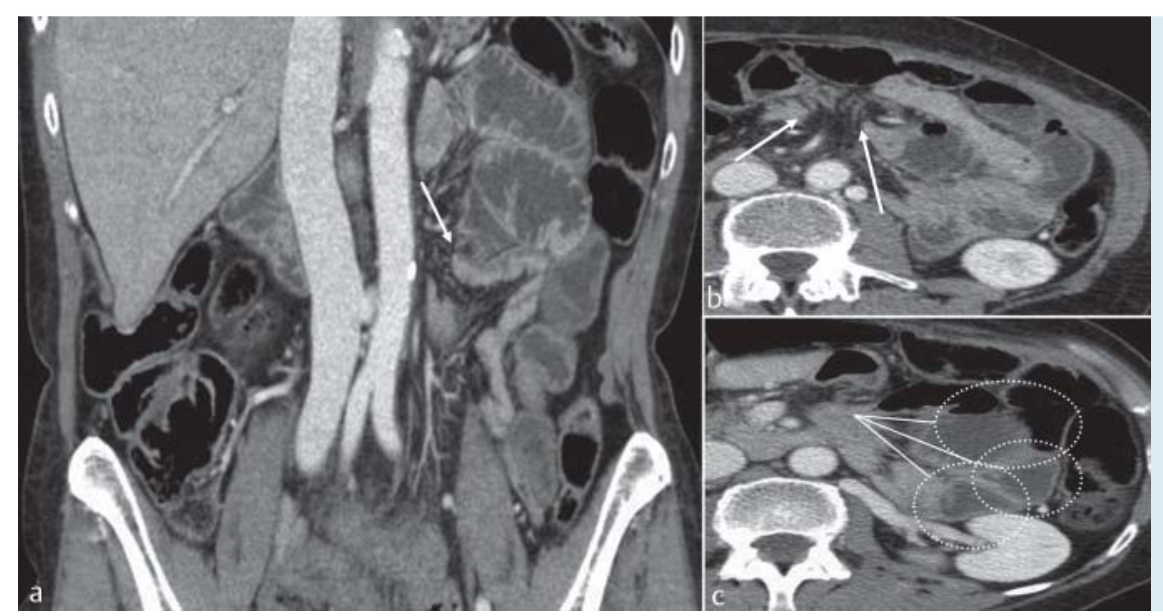

Abb.7 a-c Closed-loop-Obstruction im Rahmen einer transmesokolischen Hernie. Segmentaler Dünndarmileus (Cloosed-loop-Obstruction) betreffend die den Bruchinhalt bildenden Dünndarmschlingen a. Darstellung der analen Transitionszone (a, pfeilmarkiert) im Koronarschnitt (angulierte MPR). Im Transversalschnitt ist sowohl die orale als auch die anale Transitionszone nachweisbar (b, der Pfeil an der rechten Bruchringzirkumferenz markiert den oralen, der Pfeil linksseitig den analen Kalibersprung). „Spoke-wheel“-Sign: Die dilatierten Dünndarmschlingen sind über ihre Gefäße „speichenartig“ mit dem Bruchring verbunden (c, angulierte MPR).

\section{Lumenweite des Darmes}

Der atypische Verlauf des verlagerten Darmes mit spitzwinkeligen Angulationen im Wechsel mit gestreckten Abschnitten und die Kompression des Darmes von außen an der transmesokolischen Bruchpforte begünstigen Darmpassagestörungen. Während diffuse, vornehmlich postprandiale Schmerzen verbunden mit Nausea die Symptome einer inneren Hernie ohne Darmpassagestörung darstellen, deutet Erbrechen auf einen Ileus hin.

Dieser betrifft initial das verlagerte Darmsegment, das in der mesokolischen Bruchlücke an 2 benachbarten Stellen obstruiert wird (sog. „Closed-loop obstruction“) und in weiterer Folge den oral daran anschließenden Magen-/Darmtrakt. Somit markiert der Kalibersprung (=Transitionszone) zwischen dilatiertem Dünndarmsegment und analwärts folgendem kollabiertem Dünndarm (= "Hungerdarm“) die Lage des Bruchrings [18, 24].

\section{Lage, Kontrastmittel- und Dichteverhalten der Mesente- rien}

Bedingt durch die räumliche Nähe der den Bruchinhalt bildenden dilatierten Dünndarmschlingen, die an ihrem gemeinsamen Mesenterialgefäßstiel fixiert sind, können diese wie aufgetriebene Speichenenden eines Rades erscheinen. Die versorgenden Mesenterialgefäße konvergieren speichenartig zur Radnabe („Spoke wheel-sign“) am Bruchring [25]. Die damit einhergehende Verjüngung des Mesenterialgefäßstiels zur Bruchpforte bedingt die pilzförmige Konfiguration des verlagerten Mesenteriums („Mushroom-sign“) [16, 23]. ๑ Abb.4a-c und $\odot$ Abb. 7a-c demonstrieren anhand koronarer und axialer Reformationen die genannten Befunde.

Das Missverhältnis zwischen schmalem Bruchring und in Relation dazu langem herniertem Dünndarm begünstigt die zusätzliche Torsion dieser obstruierten Dünndarmschlingen um die Längsachse ihres Gefäßstiels, wobei der Bruchring gleichsam als Drehpunkt fungiert. Dieses „Whirl-sign“ bzw. „Swirl-sign“ wird in den Arbeiten von Lockhart et al. und Jannuccilli etal. als das wichtigste Zeichen zur Erkennung postoperativer innerer
Hernien genannt mit Sensitivitäten zwischen 74\% und $85 \%$ sowie Spezifitäten zwischen $83,3 \%$ und $86,3 \%$ bei hoher Interobserverreabilität $(\kappa>0,48)[16,23,26]$. Damit liegt neben einer möglichen mechanischen Darmpassagestörung zusätzlich eine Durchblutungsstörung vor, die - zumindest initial - nahezu ausschließlich den venösen Schenkel des intestinalen Gefäßbetts als Niederdrucksystem betrifft. Der Perfusionsdruck im arteriellen Schenkel übertrifft in der Regel den Kompressionsdruck auf das Gefäß im Rahmen der Torsion, womit die Mesenterialarterien im Gegensatz zu ihren venösen Begleitästen kontrastiert bleiben ( $\bullet$ Abb. 8b, c). Dieses Perfusionsmissverhältnis zugunsten des arteriellen Schenkels ist auch durch den Unterschied im Gefäßwandaufbau zwischen den dickwandigeren Arterien und den Venen begründet.

\section{Struktur der Darmwand}

Der Terminus Strangulationsileus subsumiert die Kombination aus Ileus und venöser Perfusionsbeeinträchtigung und weist in der Bildgebung typische Zeichen auf: Neben einer Erweiterung der Dünndarmschlingen über einen queren Außendurchmesser von $3 \mathrm{~cm}$ mit Flüssigkeits- und/oder Darmgasretention und damit möglichen klassischen Spiegelbildungen führt die Strangulation zunächst zu einer Erweiterung der dem Bruchring hepatopedal (in portovenöser Strömungsrichtung) vorgeschalteten Mesenterialvenenäste sowie zu einer Imbibierung des mesenteriellen Fettgewebes um den Gefäßstiel. In weiterer Folge kommt es zu einer Darmwandschwellung und - im Fall einer persistierenden Stase im Kapillarbett der hernierten Darmschlingen - zu deren hämorrhagischer Infarzierung. Wichtigstes Korrelat dieser intestinalen Perfusionsbeeinträchtigung ist eine verminderte Kontrastmittelaufnahme der Darmwand, die sich in einer retrospektiven Studie von Ha et al. an 84 Patienten mit einer Spezifität von $100 \%$ und einer Sensitivität von $34 \%$ als validestes Kriterium einer Strangulation herausstellte [27]. In der CT imponieren die dilatierten peripheren Mesenterialvenenäste wie Zähne eines Kammes („Comb-sign“), die intestinale Pneumatose weist auf 


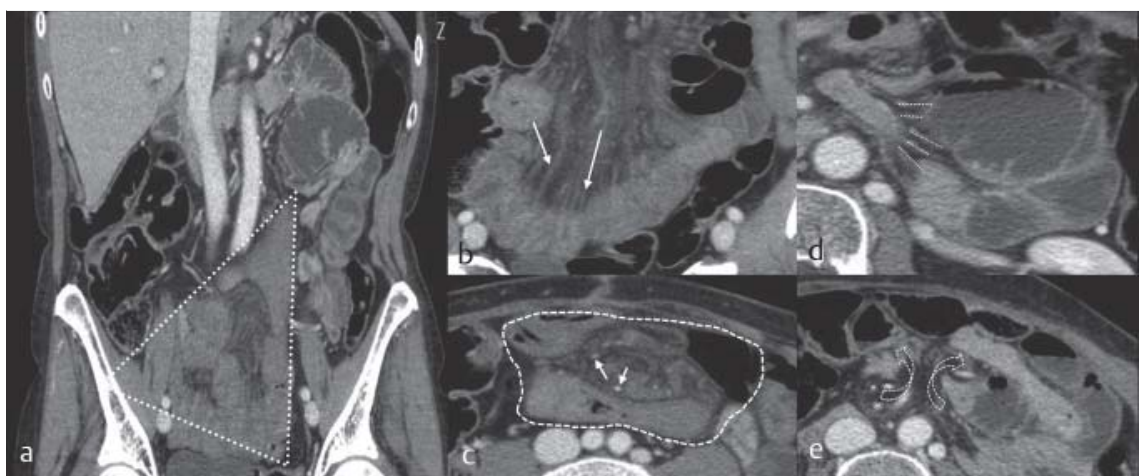

Abb. 8 a-e Kontrastmittel- und Dichteverhalten der Mesenterien sowie Struktur der Darmwand bei transmesokolischer innerer Herniation. Venöse Stauung der analen kollabierten Dünndarmschlingen mit dadurch bedingtem „Triangular sign“, das einer geometrischen Sonderform des „Creeping sign“ entspricht a. Fehlende Kontrastierung der betroffenen V. mesenterica superior-Äste (b, Pfeile). Im Gegensatz dazu sind die peripheren A.-mesenterica-superior-Äste kontrastiert (c, kurze Pfeile). Die Konvergenz des analen Dünndarmmesenteriums zur Bruchpforte führt im Axialschnitt durch den Mittelbauch zum Bild des umschriebenen verdichteten Mesenteriums umfasst von Dünndarmschlingen, das an das Auge eines Hurrikans („Hurricane eye-sign“) erinnert (c, strichlierte Linie). Durch die Stauung kommt es zu einer Erweiterung der peripheren Gekrösevenen sowie zu einer Imbibierung der Mesenterien, die sich als „Comb-sign“ in der CT manifestieren d. Die zusätzliche Torsion des mesenteriellen Gefäßstiels führt zum „Whirlsign“e.

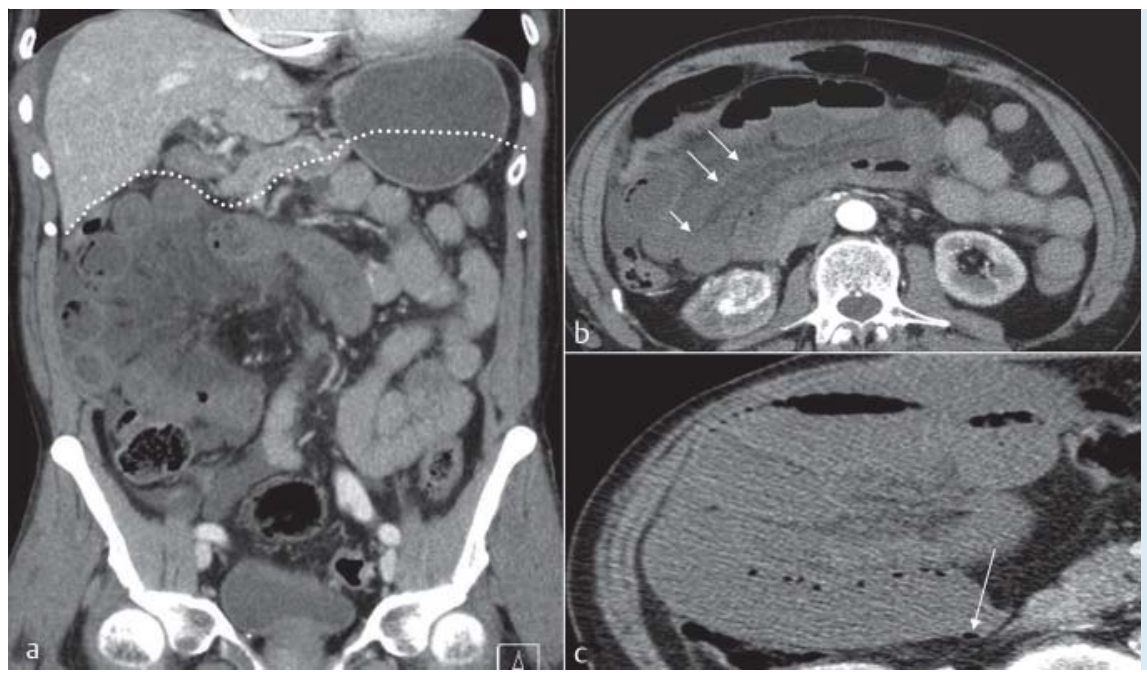

Abb.9 a-c Zur Bedeutung des Mesocolon transversum als kraniale Begrenzung des „Darmbauchs“. Typische Befunde eines segmentalen Strangulationsileus aufgrund einer Bride im rechten Mittelbauch. Im Gegensatz zu einer transmesokolischen inneren Hernie liegen die dilatierten Dünndarmschlingen kaudal der Mesocolon-transversum-Ansatzlinie (a strichlierte Linie). Fehlende Kontrastierung der strangulierten Dünndarmschlingen, hinweisend auf eine schwerwiegende Perfusionsstörung (b, Pfeile). Histologisch gesichertes Nierenzellkarzinom rechts kaudal als Nebenbefund b. Inzipiente intestinale Pneumatose (c, Pfeil): Der dorsale Gaseinschluss ist eindeutig von aufsteigenden Lufteinschlüssen im Chymus zu differenzieren.

die hämorrhagische Infarzierung des strangulierten Darmes mit nachfolgender Besiedelung durch gasbildende Darmbakterien hin [24, 27].

Weitere klassische Befunde des segmentalen Strangulationsileus in der CT sind das „Peak-sign“ bzw. das „Triangular-sign“, wobei die Spitzen des Schnabels bzw. des Dreiecks zum Bruchring weisen ( $\bullet$ Abb. 8a). Diese beiden Zeichen sind Folge der Gekröseimbibierung und damit Sonderformen des „Creeping fat-signs“ [24]. Das „Hurricane eye-sign“ ist ebenso Folge der Konvergenz des torquierten, imbibierten analen Dünndarmmesenteriums zur Bruchpforte, wodurch sich in axialen Schichten durch den Mittelbzw. Unterbauch das Bild eines runden oder ovalen verdichteten Mesenteriums ergibt, das von Dünndarmschlingen umgeben ist [16, 23].

- Abb. 7, 8 illustrieren die letztgenannten Zeichen, wobei anzumerken ist, dass im gezeigten Fall trotz Torsion nur eine geringfügige venöse Stauung des Darmes bestand.

Neben einem verminderten Kontrastmittelenhancement der Darmwand weisen laut der Arbeit von Ha et al. eine gezackte, schnabelförmige Konfiguration der Transitionszone, große Mengen freier intraperitonealer Flüssigkeit, Unschärfe der Mesente- rien sowie Dilatation der Mesenterialvenen bei gleichzeitig atypischem Verlauf auf eine Strangulation hin [27]. Die CT hat somit auch eine gewisse prognostische Bedeutung, wenngleich die Beurteilung der Darmvitalität ausschließlich mittels CT zu unsicher ist, hier ist die chirurgische Exploration unangefochtener Goldstandard [28].

Die Tab.3, 4 fassen einige wichtige diagnostische Zeichen postoperativer innerer Hernien in der CT zusammen.

\section{Differenzialdiagnostisch relevante Entitäten $\nabla$}

Mit bisweilen nahezu identischen diagnostischen Zeichen in der CT stellen Briden die wichtigste differenzialdiagnostische Entität dar, die zu einem segmentalen Strangulationsileus führen kann. Abgesehen von einer Ausnahme sind sämtliche beschriebenen CT-Befunde innerer Hernien auch bei einem Strangulationsileus zu beobachten, der durch eine Bride verursacht wird. Diese Ausnahme ist die unterschiedliche Lage der hernierten Dünndarmschlingen: Nur im Fall einer transmesokolischen Hernie überschreiten diese die Ebene der Ansatzlinie des Mesocolon 
Tab.3 Diagnostische Zeichen innerer Hernien nach laparoskopischer RouxY-Rekonstruktion in der CT. In dieser Tabelle werden Zeichen angeführt, die auf sämtliche Formen postoperativer innerer Hernien anwendbar sind. Die angegebenen Sensitivitäten und Spezifitäten sind Durchschnittswerte nach Lockhart et al. und lannuccilli et al., ermittelt an insgesamt 27 Patienten durch jeweils 3 Befunder [15, 22].

\begin{tabular}{|llc|}
\hline diagnostisches Zeichen & $\begin{array}{l}\text { Sensitivitäten in } \\
{[\mathbf{1 5 , 2 2}] \text { in \% }}\end{array}$ & $\begin{array}{c}\text { Spezifitäten in } \\
{[\mathbf{1 5}, \mathbf{2 2}] \text { in \% }}\end{array}$ \\
\hline Whirl-Sign & $\mathbf{7 4 / 8 5}$ & $83,3 / 86,3$ \\
\hline Mushroom-Sign & $46 / 25,9$ & $92,6 / 100$ \\
\hline Hurricane-Eye-Sign & $11,3 / 48,1$ & $100 / 86,6$ \\
\hline Clustered loops & $9,6 / 29,6$ & $77,6 / 80$ \\
\hline
\end{tabular}

Tab.4 Diagnostische Zeichen innerer Hernien nach laparoskopischer RouxY Rekonstruktion in der CT. In dieser Tabelle sind einige der von Reddy et al. für postoperative transmesokolische Hernien beschriebenen Zeichen inkl. Häufigkeitsangaben zusammengefasst. Auch diese Untersuchung umfasste 27 Patienten [13].

\begin{tabular}{ll}
\hline diagnostisches Zeichen & $\begin{array}{l}\text { Häufigkeiten } \\
\text { in \% }\end{array}$ \\
$\begin{array}{l}\text { kranial des Mesocolon transversum liegende Dünndarm- } \\
\text { schlingen }\end{array}$ & 100 \\
\hline $\begin{array}{l}\text { Kranialverlagerung der Jejunojejunostomie } \\
\text { in das linke Epigastrium verlagerte Mesenterialgefäßäste }\end{array}$ & 96 \\
\hline $\begin{array}{l}\text { 'Die Jejunojejunostomie sollte das Niveau des mittleren Nierendrittels nach kranial hin } \\
\text { nicht überschreiten. }\end{array}$
\end{tabular}

Tab. 5 Checkliste zur bildgebenden Diagnostik transmesokolischer innerer Hernien nach retrokolischer Roux-Y-Rekonstruktion.

\section{C: typische Befunde transmesokolischer Hernien}

\begin{tabular}{|c|c|c|}
\hline \multicolumn{3}{|c|}{ A: obligat aufzusuchende Landmarken u. deren Lokalisation bzw. Topographie } \\
\hline \multicolumn{3}{|c|}{ Gastrointestinum $\quad$ Gefäße $\quad$ Mesenterien } \\
\hline Magen im linken Epigastrium & Vasa mesenterica superiora u. deren To- & Mesocolon transversum: \\
\hline Treitz-Flexur im linken Epigastrium & pografie: & - markiert durch A. u. V. colica media \\
\hline Coecum im rechten Hypogastrium & - VMS rechts der AMS, im rechten & Ansatzlinie d. Mesocolon transversum: \\
\hline & $\begin{array}{l}\text { - Hypogastrium als Vasa iliocolica en- } \\
\text { dend }\end{array}$ & $\begin{array}{l}\text { - dem Unterrand des Pankreaskörpers } \\
\text { bzw. -schwanzes folgend }\end{array}$ \\
\hline & A. colica media: aus AMS abgehend & Dünndarmmesenterium: \\
\hline & V. colica media: in VMS drainierend & - markiert durch AMS- bzw. VMS- \\
\hline & VMI: drainierend in V. lienalis, in Lanzert- & Hauptstamm \\
\hline & $\begin{array}{l}\text { Falte verlaufend; alternativ in VMS ein- } \\
\text { mündend }\end{array}$ & \\
\hline
\end{tabular}

\section{B: abnorme CT-Befunde}

\section{Lage des Darmes}

Atypische Lage u. Zusammenbal- Dorsal der AMS liegende Dünndarm-

lung von Darmschlingen? „Radspei- schlingen (außer Pars horizontalis duo-

chen-Zeichen"? deni)?

Räumliche Beziehung der Darmschlingen zur Ansatzlinie d. Mesocolon transversum?

Verdrängung von Darmschlingen?

Konglomerat von Dünndarmschlingen im linken Epigastrium kranial der Abrisslinie des Mesocolon transversum Struktur der Darmwand

(Konzentrische) Verdickung? Vermindertes KM-Enhancement? Wandunschärfe?

Dichteanhebung nativ?

Pneumatose?

Konzentrisch verdickte, nativ dichteangehobene Darmwand mit vermindertem KM-Enhancement u. unscharfer Außenkontur

Lumenweite des Darmes

(Segmentale) Dilatation?

Dilatation d. Magens u. oralen

Dünndarms?

Poststenotischer Hungerdarm?

Segmentaler Dünndarmileus.

Evtl. auch Dilatation d. Magens u. oralen Dünndarms, poststenotischer Hungerdarm

Lage, Kontrastmittel- $u$. Dichteverhalten der Mesenterien

Mesenteriumverlagerung? Korres- Verlagerung der im jeweiligen Mesente- Röntgendichte?:

pondierend zu Darmschlingen?

rium verlaufenden Gefäße?

- Hinweis auf venöse Stauung?

Konvergenzzeichen?

- Gekröseödem?

Torquierungshinweis?

- Intermesenteriale Flüssigkeit?

In das linke Epigastrium verlagerte, imbibierte, torquierte Mesenterien mit Konvergenz zur Bruchpforte

Atypische Lage und Verlauf der Mesenterialgefäße

C: typische Befunde transmesokolischer innerer Hernien

transversum nach kranial hin. $\bullet$ Abb. 9a, b veranschaulichen dieses wichtige differenzialdiagnostische Kriterium und verdeutlichen die Bedeutung des Mesocolon transversum als kraniale Grenze des „Darmbauchs“. Da die Prävalenz peritonealer Adhä- sionen die der inneren Hernien bei Weitem übertrifft, müssen sämtliche genannten diagnostischen Kriterien, insbesondere die pathognomonische Lage der dilatierten Darmschlingen kranial der Mesocolon-transversum-Ansatzlinie gegeben sein, um eine 


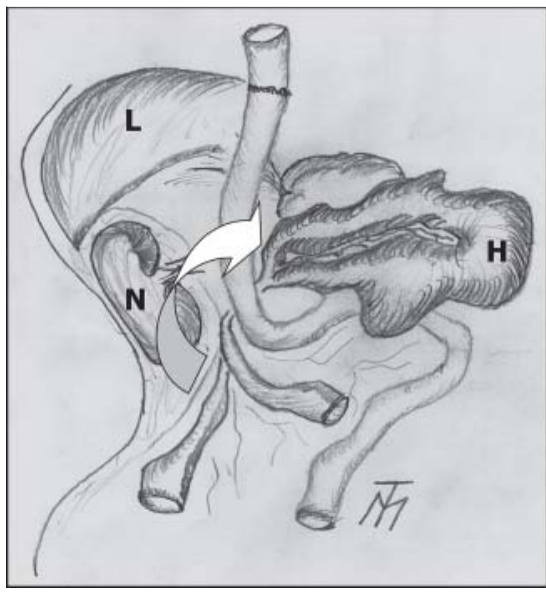

Abb. 10 PetersenHernie schematisch. Der gebogene Pfeil stellt die Richtung der Dünndarmherniation dar. Ansicht von ventral. Hernie (H), Leber (L), rechte Niere (N).

innere Hernie diagnostizieren zu können. Andernfalls ist in erster Linie von einer Bride als Ursache des segmentalen Dünndarmileus auszugehen [29]. Die $\mathbf{A b b . 9 c}$ zeigt eine inzipiente intestinale Pneumatose bei einem durch eine Bride verursachten Strangulationsileus.

Aufgrund der heute favorisierten laparoskopischen Operationstechnik gewinnen die Petersen-Hernien zunehmend an Bedeutung ( $\bullet$ Abb. 10). Die Bruchlücke wird durch den oft schmalen Spalt zwischen dem (viszeralen) Mesenterium der hochgezogenen Jejunumschlinge und dem parietalen Peritoneum bzw. dem Mesocolon transversum gebildet. Lockhart et al., Comeau et al. und Carmody et al. konnten Petersen-Hernien als häufigsten Hernientyp nach laparoskopischem Magenbypass nachweisen [16, 30, 31]. Sowohl nach retrokolischer als auch nach antekolischer Elevation des Jejunums können Petersen-Hernien auftreten. Der antekolische Hochzug wird in der bariatrischen Chirurgie zunehmend häufiger eingesetzt, um die Entstehung einer transmesokolischen Hernie zu verhindern [11].

Der fehlende peritoneale Bruchsack erlaubt bei orthotoper Lage der intestinalen Landmarken die Abgrenzung von kongenitalen inneren Hernien, da diesen nicht selten Malrotationen zugrunde liegen [18].

\section{Zusammenfassung}

Die systematische Analyse einer CT-Studie des Magendarmtrakts basierend auf der obligaten Beurteilung intestinaler, vaskulärer und vor allem mesenterieller Landmarken ermöglicht die Diagnose innerer Hernien.

Die Erkennung postoperativer innerer Hernien erfordert profunde Kenntnisse der Schnittbildanatomie und der Operationsanamnese.

Nachfolgende Checkliste ist als Hands-on für die tägliche Praxis konzipiert ( $\bullet$ Tab.5).

\section{Literatur}

1 Riemann JF, Fischbach W, Galle PR et al. (eds) Gastroenterologie. Stuttgart: Thieme; 2010, 521-543

2 Grenacher L, Schwarz M, Lordick F et al. Aktualisierte S3 - Leitlinie zur Diagnostik und Therapie des Magenkarzinoms: Bedeutung für die Radiologische Diagnostik. Fortschr Röntgenstr 2012; 184: 706-712

3 Livingston EH. Obesity and its surgical management. The American Journal of Surgery 2002; 184: 103-113
4 Gastrointestinal surgery for severe obesity: National Institutes of Health consensus development conference statement. Am J Clin Nutr 1992; 55: 615S-619S

5 Sandrasegaran K, Rajesh A, Lall C et al. Gastrointestinal complications of bariatric Roux-en-Y gastric bypass surgery. Eur Radiol 2005; 15: $254-262$

6 DeMaria EJ, Sugerman HJ, Kellum JMK et al. Results of 281 consecutive total laparoscopic Roux-en-Y gastric bypasses to treat morbid obesity. Ann Surg 2002; 235: 640-647

7 Schauer PR, Ikramuddin S, Gourash W et al. Outcomes after laparoscopic Roux-en-Y gastric bypass for morbid obesity. Ann Surg 2000; 232 : $515-529$

8 Scheirey CD, Scholz FJ, Paresh CS et al. Radiology of the laparoscopic Roux-en-Y gastric bypass procedure: conceptualization and precise interpretation of results. Radiographics 2006; 26: 1355 -1371

9 Higa $K D$, Boone $K B$, Ho T et al. Laparoscopic Roux-en-Y gastric bypass for morbid obesity. Technique and preliminary results of our first 400 patients. Arch Surg 2000; 135: 1029-1033

10 Thiele J, Vothel F, Scheibe J. Hernia obturatoria - ein seltener Eingeweidebruch. Fortschr Röntgenstr 2011; 183: 393 - 394

11 Ahmed AR, Richards G, Husain S et al. Trends in internal hernia after laparoscopix Roux-en-Y gastric bypass. Obes Surg 2007; 17: 1563 1566

12 Blachar A, Federle MP. Gastrointestinal complications of laparoscopic roux-en-Y bypass surgery in patients who are morbidly obese: findings on radiography and CT. Am J Roentgenol 2002; 179: 1437-1442

13 Neumeister B, Besenthal I, Böhm BO. Klinikleitfaden Labordiagnostik Elsevier Urban \& Fischer Verlag; 2009, ISBN 978-3-437-22232-090 92,224

14 Egger L, Fikentscher T, Poschenrieder F et al. „Klinischer Stellenwert der Durchleuchtung der Magen-Darm-Passage (MDP) an einem Universitätsklinikum. Fortschr Röntgenstr 2012; 184: 893-898

15 Reddy SA, Yang C, McGinnis LA et al. Diagnosis of transmesocolic internal hernia as a complication of retrocolic gastric bypass: CT imaging criteria. Am J Roentgenol 2007; 189: 52-55

16 Lockhart ME, Tessler FN, Canon CL et al. Internal hernia after gastric bypass: sensitivity and specifity of seven CT signs with surgical correlation and controls. Am J Roentgenol 2007; 188: 745 - 750

17 Merkle EM, Hallowell PT, Crouse C et al. Roux-en-y gastric bypass for clinically severe obesity: normal appearance and spectrum of complications at imaging. Radiology 2005; 234: 674-683

18 Takeyama N, Gokan T, Ohgiya Y et al. CT of internal hernias. Radiographics 2005; 25: $997-1015$

19 Martin L, Merkle EM, Thompson WM. Review of internal hernias: radiographic and clinical findings. Am J Roentgenol 2006; 186: 703 - 717

20 Okino Y, Kiyusue H, Mori H et al. Root of small-bowel mesentery: correlative anatomy and CT-features of pathologic conditions. Radiographics 2001; 21: $1475-1490$

21 Stadler T, Langman J. Medizinische Embryologie. Die normale menschliche Entwicklung und ihre Fehlbildungen. Stuttgart: Thieme; 2003, $248-273$

22 Hafferl A, Thiel W. Lehrbuch der topographischen Anatomie. Stuttgart: Springer; 1969, 440-448, 503-534

23 Iannuccilli JD, Grand D, Murphy BL et al. Sensitivity and specificity of eight CT signs in the preoperative diagnosis of internal mesenteric hernia following Roux-en-Y gastric bypass surgery. Clin Radiol 2009; 64: $373-380$

24 Balthazar EJ, Birnbaum B, Megibow AJ et al. Closed-loop and strangulation intestinal obstruction: CT-signs. Radiology 1992; 185: 769 - 775

25 Rudloff $U$. The spoke wheel sign: bowel. Radiology 2005; 237: 1046 1047

26 Khurana $B$. The whirl sign. Radiology 2003; 226: 69-70

27 Ha HK, Kim JS, Lee MS. Differentiation of simple and strangulated small bowel obstructions: usefulness of known CT criteria. Radiology 1997; 204: $507-512$

28 Hwang JY, Lee JK, Lee JE et al. Value of multidetector CT in decision making regarding surgery in patients with small-bowel obstruction due to adhesion. Eur Radiol 2009; 19: 2425 - 2431

29 Meyers MA. Paraduodenal hernias: radiologic and arteriographic diagnosis. Radiology 1970; 95: 29-37

30 Comeau E, Gagner M, Inabnet WB et al. Symptomatic internal hernias after laparoscopic bariatric surgery. Surg Endosc 2005; 19: 34- 39

31 Carmody B, DeMaria EJ, Jamal M et al. Internal hernia after laparoscopic RouX-en-Y gastric bypass. Surg Obes Relat Dis 2005; 6: 543-548 